\title{
A Strategy for Using Intraoperative Nerve Monitoring During Esophagectomy to Prevent Recurrent Laryngeal Nerve Palsy
}

\author{
MASAMI YUDA, KATSUNORI NISHIKAWA, KEITA TAKAHASHI, TAKANORI KUROGOCHI, YUJIRO TANAKA, \\ AKIRA MATSUMOTO, YUICHIRO TANISHIMA, NORIO MITSUMORI and KATSUHIKO YANAGA
}

Department of Surgery, The Jikei University School of Medicine, Tokyo, Japan

\begin{abstract}
Background: There have been only sporadic reports on intraoperative nerve monitoring (IONM) during esophageal cancer surgery. We aimed to establish a strategy for the use of IONM during esophagectomy. Patients and Methods: Fortyone cases enrolled in this study. The IONM was performed before and after lymph node dissection in the thoracic cavity and cervical area. Occurrence of recurrent laryngeal nerve $(R L N)$ palsy was assessed on the seventh postoperative day. Results: The identification of nerves using IONM was possible in all cases. The positive and negative predictive values of IOMN were $80 \%$ and $92 \%$, respectively. Loss of response was observed during the thoracic procedure in 14 out of 16 cases, with the predominance of left $R L N$ palsy $(n=12)$. Conclusion: In esophageal cancer surgery, identification of the RLN using IONM can be carried out safely, simply, and promptly. Using IONM systematically, the prediction of RLN palsy and detection of nerve injury point seems feasible.
\end{abstract}

Recurrent laryngeal nerve (RLN) palsy can occur as a consequence of surgical treatment of esophageal cancer, and may result in a motility disorder of the vocal cord muscles, causing hoarseness of the voice or occasionally fatal respiratory complications $(1,2)$. The frequency of RLN palsy following esophageal cancer surgery has been reported to range from $15.6 \%$ to $80 \%$ (1-6), for which the main cause is the failure to recognize a perioperative neural injury. Despite this risk during surgery, there is currently no established method to monitor patients for perioperative injury of RLN that could result in RLN palsy.

The technique of nerve monitoring has been clinically applied during thyroid surgery and neurosurgery since the 1960s and 1970s, respectively (7-9). Recently, the use of intraoperative

Correspondence to: Masami Yuda, MD, Department of Surgery, Jikei University School of Medicine, 3-25-8 Nishi-shinbashi, Minato-ku, Tokyo, 105-8461, Japan. E-mail: my99092jp@yahoo.co.jp

Key Words: Esophageal cancer, esophagectomy, intraoperative nerve monitoring, recurrent laryngeal nerve, vagus nerve. nerve monitoring (IONM) for the purpose of nerve identification during thyroid surgery has been reported to reduce the incidence of RLN palsy (10-16). The IONM procedure involves attaching an intubation tube with a surface electrode to the vocal cord muscles and electrically stimulating the RLN. This results in visual and aural signals of electromyographic activity. To date, there have been only sporadic reports on IONM during esophageal cancer surgery (17-21).

The aim of this study was to investigate the effects of RLN stimulation using a nerve stimulator during thoracic and cervical procedures of esophageal cancer surgery. From these results, the study aimed to establish an original strategy regarding the use of IONM during esophageal cancer surgery to reduce postoperative vocal cord palsy.

\section{Patients and Methods}

We enrolled 41 cases with esophageal cancer who underwent IONM, with an overall median age of $63.0 \pm 7.1$ years, of whom $38(93 \%)$ were male (Table I). This study was carried out at the Department of Surgery at the Jikei University School of Medicine between January 2011 and December 2014. All patients underwent an esophagectomy with three-field lymph node dissection, either via right thoracotomy or under a thoracoscope for thoracic esophageal carcinoma. The current study was approved by the Hospital Ethics Committee of Jikei University School of Medicine (Approval number: 28-054 8297), and written informed consent was obtained from all patients undergoing IONM. Fifteen out of 56 patients were excluded from the study because of electrode disconnection.

A single lumen intubation tube (TriVantage EMG tube; Medtronic, Tokyo, Japan) specifically designed for a nerve integrated monitoring system (NIM Response 2.0; manufactured by Medtronic, Tokyo, Japan) was routinely inserted under general anesthesia. The electrode was fixed to the vocal cord. Esophagectomy and lymph node dissection were performed under single left lung ventilation with a right endobronchial blocker by well-trained esophageal surgeons. Esophagectomy and intrathoracic lymph node dissection including the regional lymph nodes adjacent to RLN were carried out in the left lateral decubitus position. Subsequently, reconstruction with a gastric tube was carried out after changing the posture to the supine position.

Figures 1 and 2 show the intraoperative images during nerve stimulation. When the stimulation probe contacts the RLN, electrical stimulation causes contraction of the vocal cord muscles which can be 
recognized by the electromyogram. This procedure enables pinpoint nerve stimulation because the probe tip is very small $(0.33 \mathrm{~mm})$ and surrounded by effective insulation. The standard current of electrical stimulation was $1.0 \mathrm{~mA}$.

Occurrence of RLN palsy was assessed on the seventh postoperative day by observation of vocal cord movement at vocalization using transnasal endoscopy by a surgeon or an otolaryngologist.

Procedure of IONM. Thoracic procedure. Identification of the RLN: Before upper mediastinum lymph node dissection, the RLN was identified by observation of any contraction of the vocal cord muscles with electrical nerve stimulation.

Confirmation of the viability of the RLN following dissection: After the upper mediastinum lymph node dissection, the RLN was again stimulated on the most proximal side of the dissected area to confirm the viability of the RLN.

Cervical procedure. Identification of the RLN/vagus nerve (VN): Prior to cervical lymph node dissection, VN was identified by observation of any contraction of the vocal cord muscles with electrical nerve stimulation. Similarly to the thoracic procedure, the RLN was identified by observation of any contraction of the vocal cord muscles with electrical nerve stimulation.

Confirmation of the viability of the RLN following dissection: After completion of the cervical lymph node dissection, electrical stimulation of the RLN was applied to the most proximal side of the lymph node dissected area. Electrical stimulation of the $\mathrm{VN}$ was also applied to check the contraction of the vocal cord muscles.

When the RLN was damaged during IONM, the monitoring amplitude of electrical voltage decreased. The loss of response (LOR) was defined as no amplitude during IONM stimulation, which was recorded during the procedure described above.

The endpoint of this study was to establish the strategy for using IONM during esophageal cancer surgery by measurement of positive and negative predictive value of IONM.

\section{Results}

Regardless of the patient demographics shown in Table I, the identification of nerves using IONM was possible in all cases. The stimulation probe was able to contact the nerve through the thoracoscopic port or directly in the open thoracotomy procedure. No adverse consequences of IONM were observed during a mean follow-up period of 13.4 months.

Postoperative RLN palsy was observed in one case (2\%) on the right side, in eight cases $(20 \%)$ on the left side, and in six cases $(15 \%)$ on both sides. Table II shows the results of LOR observed during surgery along with the incidence of postoperative RLN palsy. The probability (positive predictive value, PPV) of LOR being observed during surgery along with the occurrence of postoperative RLN palsy was $80 \%$. Conversely, the probability (negative predictive value, NPV) of LOR not being observed during surgery and postoperative RLN palsy not being found was $92 \%$.

Counting the patient with bilateral RLN palsy as two cases, there was difficulty in identifying the nerve injury in five out
Table I. Patient characteristics.

\begin{tabular}{lc}
\hline Characteristic & $\mathrm{n}=41$ \\
\hline Age (years), mean \pm SD & $63.0 \pm 7.14$ \\
Gender, $\mathrm{n}(\%)$ & $38(93 \%)$ \\
$\quad$ Male & $3(7 \%)$ \\
Female & \\
Location of cancer, $\mathrm{n}(\%)$ & $6(15 \%)$ \\
Upper esophagus & $19(46 \%)$ \\
Middle esophagus & $16(59 \%)$ \\
Lower esophagus & \\
Type of operation, $\mathrm{n}(\%)$ & $25(61 \%)$ \\
Right-thoracotomy & $16(39 \%)$ \\
Thoracoscopic & \\
Clinical stage, $\mathrm{n}(\%)$ & $17(42 \%)$ \\
I & $10(24 \%)$ \\
II & $14(34 \%)$ \\
III & 0 \\
IV & \\
Metastasis to the lymph node around RLN, $\mathrm{n}$ & $4: 37$ \\
Right side: Yes/no & $3: 38$ \\
Left side: Yes/no & $16(39 \%)$ \\
Neoadjuvant chemotherapy, $\mathrm{n}(\%)$ & $25(61 \%)$ \\
No & \\
Yes &
\end{tabular}

Based on the seventh edition of the TNM staging of Union for International Cancer Control.

Table II. Result of intraoperative nerve monitoring.

\begin{tabular}{lccllc}
\hline & \multicolumn{4}{c}{ Intraoperative LOR, $\mathrm{n}$} \\
\cline { 2 - 3 } & \multicolumn{2}{c}{ Left RLN/VN } & & \multicolumn{2}{c}{ Right RLN/VN } \\
\cline { 2 - 3 } \cline { 5 - 6 } Nerve palsy & Yes & No & & Yes & No \\
\hline Yes & 12 & 2 & & 4 & 3 \\
No & 4 & 23 & & 0 & 34 \\
Total & 16 & 25 & & 4 & 37 \\
\hline
\end{tabular}

RLN: Recurrent laryngeal nerve, VN: vagus nerve, LOR: loss of response. Positive predictive value: 16 (palsy) out of 20 (nerves) $=80 \%$, negative predictive value: 57 (palsy) out of 62 (nerves) $=91.9 \%$.

Table III. Location of loss of response (LOR) in patients with postoperative nerve palsy.

\begin{tabular}{lcc}
\hline & \multicolumn{2}{c}{ Nerve palsy, $\mathrm{n}$} \\
\cline { 2 - 3 } Location of LOR & Left RLN/VN & Right RLN/VN \\
\hline Cervical area & 0 & 2 \\
Thoracic cavity & 12 & 2 \\
Undetermined & 2 & 3 \\
\hline
\end{tabular}

RLN: Recurrent laryngeal nerve, VN: vagus nerve. 


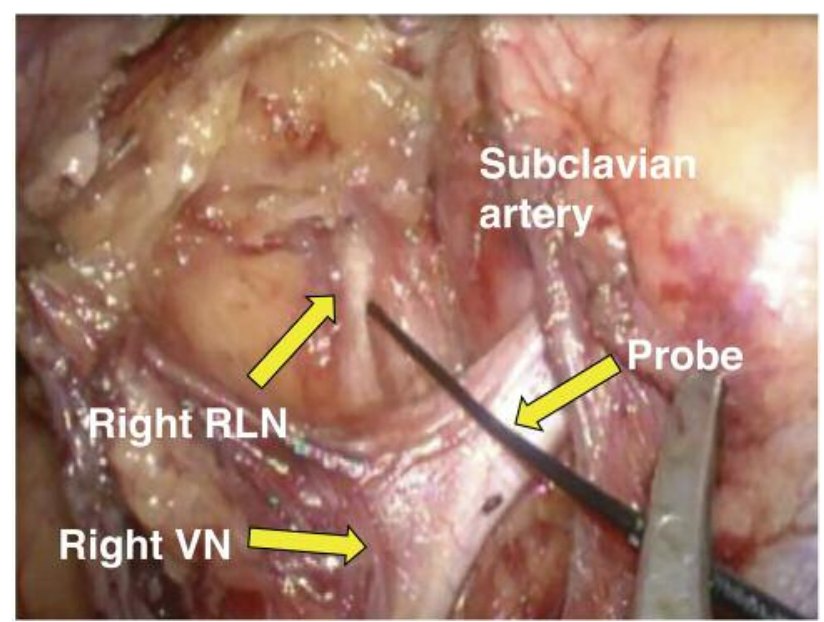

Figure 1. Intraoperative recurrent laryngeal nerve (RLN) stimulation by an intraoperative nerve monitoring probe in the right thoracic cavity. A fine stimulation probe can easily contact the RLN without causing any damage to the RLN or adjacent organs. The surrounding anatomical structures of the RLN are labeled. VN: Vagus nerve.

of the 21 cases of postoperative RLN palsy because LOR was not observed in either the thoracic cavity or the cervical areas (Table III). Out of the remaining 16 cases, LOR was confirmed during the thoracic procedure in $14(88 \%), 12$ $(86 \%)$ of which were of left RLN palsy. Both patients with LOR only in the cervical region had right RLN palsy.

\section{Discussion}

Thoracic esophageal carcinoma most frequently metastasizes to the upper mediastinal lymph nodes close to RLN. Therefore, dissection of those lymph nodes is an important maneuver for curative esophageal cancer surgery (5). To prevent fatal respiratory complications after esophagectomy, countermeasures to lower the risk of developing postoperative RLN palsy are very important. IONM is intended to identify nerves safely and easily during surgery, avoiding injury and lowering the risk of RLN palsy. Although there are many reports that IONM is useful in thyroid surgery (10-16), only a few studies of IONM use have been reported in esophageal cancer surgery. This is generally due to the anatomical constraints associated with upper mediastinal lymph node dissection close to the RLN in the intrathoracic cavity during esophageal cancer surgery. Unlike thyroid gland and brain surgery where IONM is more frequently used, esophageal cancer surgery is handicapped by the posture of the patient and the logistics involved in stimulating the nerve in the limited space of the intrathoracic cavity. Moreover, conducting esophagectomy during right thoracotomy requires the maintenance of single lung ventilation in the left lateral

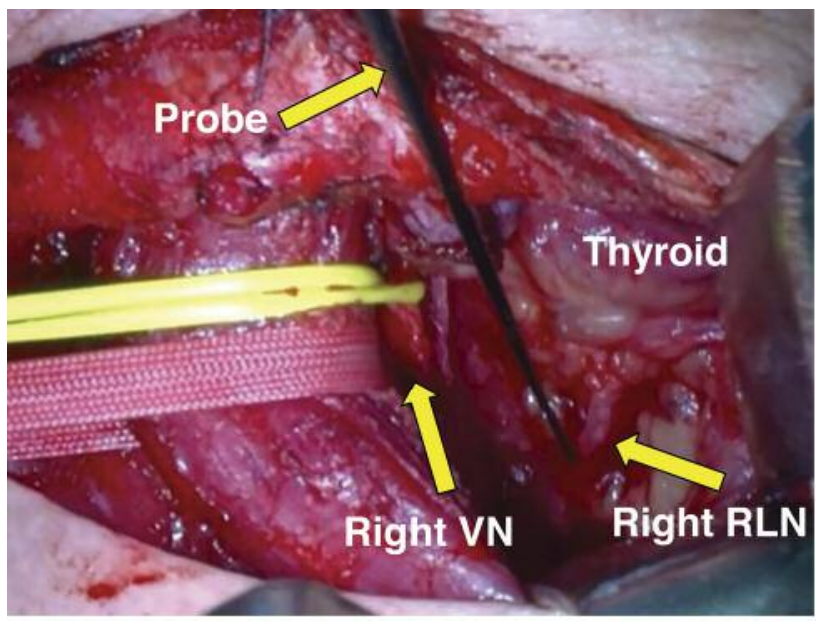

Figure 2. Intraoperative recurrent laryngeal nerve (RLN) stimulation by an intraoperative nerve monitoring probe in the right cervical area. In contrast to the thoracic procedure, the stimulation of both the RLN and vagus nerve $(V N)$ can be performed during cervical lymphadenectomy. The surrounding anatomical structures of the RLN are labeled.

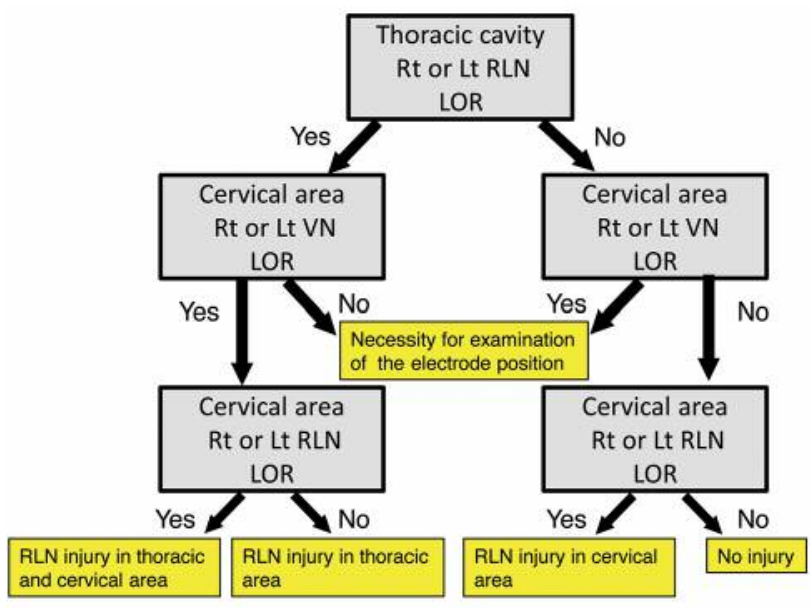

Figure 3. A flow chart of the strategy for using an intraoperative nerve monitoring system during esophagectomy. LOR: Loss of response; Lt: left; RLN: recurrent laryngeal nerve; Rt: right; VN: vagus nerve.

decubitus position, resulting in difficulty in maintaining the electrode of the tube in contact with the vocal cords. There is also difficulty in maintaining contact of the electrode after changing postures. Despite these difficulties, Hemmerling et al. reported that IONM is possible during esophageal cancer surgery by adjusting the angle of the contact electrode of the conventional double lumen tube (17). In addition, Ikeda et al. reported that IONM is possible using the same single lumen TriVantage EMG tube during thoracoscopic surgery in the prone position (18). In the current study, we found that 
positional adjustment of the electrode was feasible after changing the patient to the supine position.

Regarding the exclusion of 15 cases from this study due to inability to observe contraction of the vocal cord muscles during the RLN identification phase, this failure seems to have been due to poor contact of the electrode with the vocal cord or errors in setting the threshold of the nerve stimulator.

We believe that the merits of using IONM during esophageal cancer surgery are two-fold: (i) allowing safe, simple and prompt identification of the RLN; and (ii) providing ongoing surveillance to evaluate neurological function that cannot be determined visually.

Although there were some occasions during the current study on which visible identification of the RLN by the operator was difficult, it was possible to identify the RLN with certainty using IONM. The immediate identification of the RLN makes it possible to dissect lymph nodes more safely without surgeon stress. Enabling prompt perioperative identification of the RLN is of great benefit, not only at institutions where cases of esophageal cancer surgery are rare, but also at institutions where thoracoscopic surgery has just been introduced, or in training young surgeons at high-volume institutions.

Moreover, preoperative chemotherapy or chemoradiotherapy is becoming a standard treatment for advanced esophageal cancer. This preoperative treatment may cause inflammation, edema, and fibrosis of the periesophageal tissues, resulting in difficulty in the identification of RLN. Moreover, an anomaly of the RLN or subclavian artery, for example non-reccurent type, may lead to failure to identify the RLN (21-22). For such circumstances, IONM may be an effective navigation tool.

It is impossible to visually determine injury of the RLN during surgery. In this regard, IONM makes it possible to objectively evaluate the electrical activity of the nerves during surgery and thus to determine whether any injury of the RLN has occurred. Although the use of IONM in past cases for esophageal cancer surgery has been limited to RLN stimulation in the thoracic cavity (17-20), the current study also conducted stimulation of the VN in the cervical procedure. This additional check enabled evaluation of neurological function of RLN and subsequently increased the accuracy of predicting postoperative development of RLN palsy.

IONM can evaluate the activity of nerve between the site of nerve stimulation and an EMG tube. From this point of view, it is important to conduct electrical stimulation from the most proximal side of the nerve. When LOR occurs following lymph node dissection, it is possible to confirm the injured site by conducting stepped stimulation towards the peripheral side. Based on these results, we created a flow chart to illustrate the strategy regarding the use of IONM during esophageal cancer surgery (Figure 3). Unlike operations in thyroid surgery and neurosurgery, the range of lymph node dissection in esophageal cancer surgery is wide, covering both the cervical and the thoracic regions. As described above, depending on the dissection range, it is important to consider the stimulation site systematically when using IONM. We propose to follow the strategy illustrated in the flow chart for effective use of IONM and to increase the accuracy of assessment of RLN viability. In particular, it was vital to conduct stimulation of the VN prior to cervical lymph node dissection. Therefore, we conclude that IONM of the RLN functions mainly as a means of navigation, while IONM of the $\mathrm{VN}$ plays a role in predicting postoperative palsy.

In five out of the 21 cases in which palsy occurred, it could not be determined whether palsy occurred in the thoracic cavity or in the cervical area. In such cases, palsy occurred even though LOR was not observed. Ulmer et al. reported that signals received from the EMG tube are reduced by approximately $60 \%$ due to pressure and pulling of the RLN during thyroid surgery (11). In other words, even if a complete loss of electrical signals is not observed, damage to the nerves may still have occurred. It appears that the five cases with RLN palsy without LOR were damaged by the surgical procedure.

The analysis of LOR (Table III) showed that approximately $80 \%$ of LOR in the thoracic cavity occurred on the left side, while all cases in the cervical region occurred on the right side. Therefore, greater care should be paid during lymph node dissection around the left RLN in the thoracic cavity and the right RLN in the cervical area.

In the current study, muscle relaxants were used only at the beginning of the operation. However, because esophageal cancer surgery generally takes a long time, there are some occasions on which additional muscle relaxants were required. Although some studies reported the effects of various anesthetic agents during thyroid surgery on IONM (23-25), the relationship between these effects and the results of the current study are unknown.

Another limitation of this study was that it is possible that various confounding factors may have caused a bias, as the study was a retrospective one.

The predictive information on the occurrence of postoperative RLN palsy which can be obtained from IONM is important for the risk assessment in postoperative care. From this information, it may be possible to arrange the extubation timing and the start of oral intake individually. Another advantage for the use of IONM is that detection of points of injury of the RLN could highlight the surgical procedure which may cause RLN palsy. The information from IONM and review of the postoperative video recording will improve the safety of subsequent surgical procedures.

\section{Conclusion}

In esophageal cancer surgery, identification of the RLN using IONM can be carried out during either a thoracotomy or thoracoscopic surgery safely, simply, and promptly, which is 
considered to be useful not only in prevention and prediction of postoperative RLN palsy, but also for improving the safety of surgical procedures.

\section{References}

1 Gockel I, Kneist W, Keilmann A and Junginger T: Recurrent laryngeal nerve paralysis (RLNP) following esophagectmy for carcinoma. Eur J Surg Oncol 31(3): 277-281, 2005.

2 Johnson PR, Kanegoanker GS and Bates T: Indirect laryngoscopic evaluation of vocal cord function in patients undergoing transhiatal esophagectmy. J Am Coll Surg 178(6): 605-608, 1994.

3 Pertl L, Zacherl J, Mancusi G, Gachter JN, Asari R, Schoppmann S, Bigenzahn W and Schneider-Stickler B: High risk of unilateral recurrent laryngeal nerve paralysis after esophagectomy using cervical anastomosis. Eur Arch Otorhinolaryngol 268(11): 16051610, 2011.

4 Fujita H, Kakegawa T, Yamana H, Shima I, Toh Y, Tomita Y, Fujii $\mathrm{T}$, Yamasaki $\mathrm{K}$ and Noake $\mathrm{T}$ : Mortality and morbidity rates, postoperative course, quality of life and prognosis after extended radical lymphadenectomy for esophageal cancer. Ann Surg 222(5): 654-662, 1995.

5 Baba M, Aikou T, Yoshinaka H, Natsugoe S, Fukumoto T, Shimazu $\mathrm{H}$ and Akazawa K: Long-term results of subtotal esophagectomy with three-field lymphadenectomy for carcinoma of the thoracic esophagus. Ann Surg 219(3): 310-316, 1994.

6 Shimizu H, Shiozaki A, Fujisawa H, Konishi H, Kosuga T, Komatsu S, Ichikawa D, Okamoto K, Otsuji E: Short- and longterm progress of recurrent laryngeal nerve paralysis after subtotal esophagectomy. Anticancer Res 37(4): 2019-2023, 2017.

7 Flisberg K and Lindholm T: Electrical stimulation of the human recurrent laryngeal nerve during thyroid operation. Acta Otolaryngol Suppl 263: 63-67, 1969.

8 Shedd DP and Durham C: Electrical identification of the recurrent laryngeal nerve. I. Response of the canine larynx to electrical stimulation of the recurrent laryngeal nerve. Ann Surg 163(1): 4750, 1966.

9 Shimoji K, Higashi $\mathrm{H}$ and Kato T: Epidural recording of spinal electrogram in man. Electroencephalogr Clin Neurophysiol 30(3): 236-239, 1971.

10 Chiang FY, Lee KW, Chen HC, Chen HY, Lu IC, Kuo WR, Hsieh $\mathrm{MC}$ and $\mathrm{Wu} \mathrm{CW}$ : Standardization of intraoperative neuromonitoring of recurrent laryngeal nerve in thyroid operation. World J Surg 34(2): 223-229, 2010.

11 Ulmer C, Koch KP, Seimer A, Molnar V, Meyding-Lamade U, Thon KP and Lamade W: Real-time monitoring of the recurrent laryngeal nerve: an observational clinical trial. Surgery 143(3): 359-365, 2008.

12 Dralle H, Sekulla C, Lorenz K, Machens A and the German IONM Study Group: Intraoperative monitoring of the recurrent laryngeal nerve in thyroid surgery. World J Surg 32(7): 13581366, 2008.

13 Dralle H, Sekulla C, Lorenz K, Nguyen Thanh P, Schneider R and Machens A: Loss of the nerve monitoring signal during bilateral thyroid surgery. Br J Surg 99(8): 1089-1095, 2012.
14 Barczynski M, Konturek A, Pragacz K, Papier A, Stopa M and Nowak W: Intraoperative nerve monitoring can reduce prevalence of recurrent laryngeal nerve injury in thyroid reoperations: results of a retrospective cohort study. World J Surg 38(3): 599-606, 2014.

15 Phelan E, Potenza A, Slough C, Zurakowski D, Kamani D and Randolph G: Recurrent laryngeal nerve monitoring during thyroid surgery: normative vagal and recurrent laryngeal nerve electrophysiological data. Otolaryngol Head Neck Surg 147(4): 640-646, 2012.

16 White WM, Randolph GW, Hartnick CJ and Cunningham MJ: Recurrent laryngeal nerve monitoring during thyroidectomy and related cervical procedures in the pediatric population. Arch Otolaryngol Head Neck Surg 135(1): 88-94, 2009.

17 Hemmerling TM, Schmidt J, Jacobi KE and Klein P: Intraoperative monitoring of the recurrent laryngeal nerve during single-lung ventilation in esophagectomy. Anesth Analg 92(3): 662-664, 2001.

18. Ikeda Y, Inoue T, Ogawa E, Horikawa M and Fukushima R: Recurrent laryngeal nerve monitoring during thoracoscopic esophagectmy. World J Surg 38(4): 897-901, 2014.

19 Gelpke H, Grieder F, Decurtins M and Cadosch D: Recurrent laryngeal nerve monitoring during esophagectomy and mediastinal lymph node dissection. World J Surg 34(10): 2379-2382, 2010.

20 Zhong D, Zhou Y, Li Y, Wang Y, Zhou W, Cheng Q, Chen L, Zhao J, Li X and Yan X: Intraoperative recurrent laryngeal nerve monitoring: a useful method for patients with esophageal cancer. Dis Esophagus 27(5): 444-451, 2014.

21 Yamashita K, Miyata H, Kanemura T, Miyazaki Y, Makino T, Takahashi T, Kurokawa Y, Yamasaki M, Nakajima K, Takiguchi S, Mori M and Doki Y: Successful esophageal carcinoma resection with intraoperative neuromonitoring in a patient with non-recurrent inferior laryngeal nerve. Esophagus 13(1): 97-103, 2016.

22 Kitagawa H, Namikawa T, Hanazaki K: Neck Dissection and thoracoscopic esophagectomy in esophageal cancer with aberrant subclavian artery. Anticancer Res 37(7): 3787-3790, 2017.

23 Marusch F, Hussock J, Haring G, Hachenberg T and Gastinger I: Influence of muscle relaxation on neuromonitoring of the recurrent laryngeal nerve during thyroid surgery. $\mathrm{Br} \mathrm{J}$ Anaesth 94(5): 596-600, 2005.

24 Lu IC, Tsai CJ, Wu CW, Cheng KI, Wang FY, Tseng KY and Chiang FY: A comparative study between 1 and 2 effective doses of rocuronium for intraoperative neuromonitoring during thyroid surgery. Surgery 149(4): 543-548, 2011.

25 Chang PY, Wu CW, Chen HY, Chen HC, Cheng KI, Lu IC and Chiang FY: Influence of intravenous anesthetics on neuromonitoring of the recurrent laryngeal nerve during thyroid surgery. Kaohsiung J Med Sci 30(10): 499-503, 2014.

Received November 26, 2017

Revised December 22, 2017

Accepted January 3, 2018 